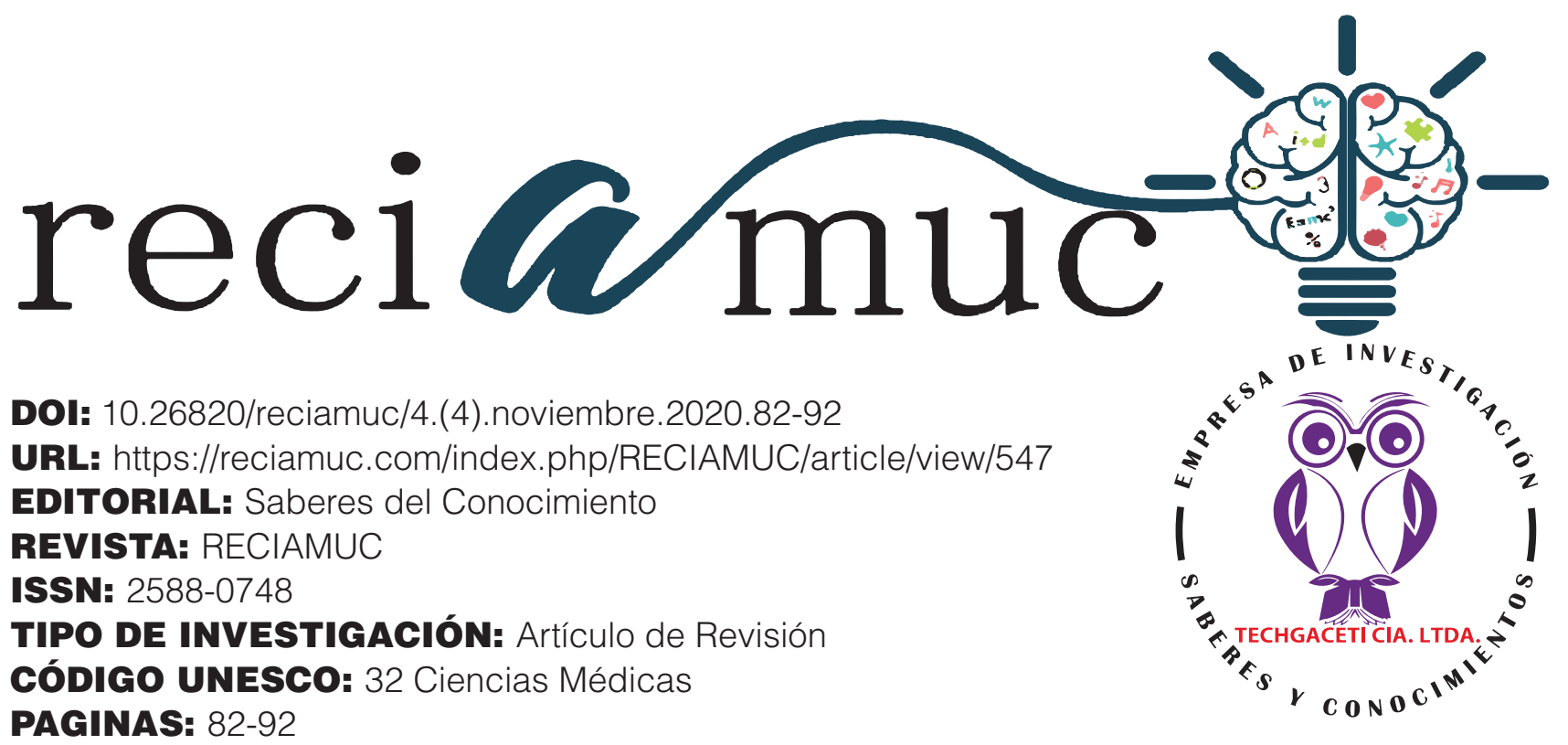

\title{
Hipertensión intracraneal idiopática, fisiopatología y manejo en pacientes en la Clínica San Francisco
}

Idiopathic intracranial hypertension, pathophysiology and management in patients at the San Francisco Clinic

Hipertensão intracraniana idiopática, fisiopatologia e manejo em pacientes na Clínica San Francisco

Pedro Enrique Decker Larrea'; Verónica Deniss Intriago Cevallos2;

Carlos Antonio Villacis Rodriguez ${ }^{3}$; Jefferson Leonardo León García ${ }^{4}$

\section{RECIBIDO: 18/07/2020 ACEPTADO: 20/09/2020 PUBLICADO: 30/11/2020}

1. Médico; Investigador Independiente; Guayaquil, Ecuador; pedro_deckerbond@hotmail.com; (D) https://orcid.org/00000003-1567-0245

2. Médico; Investigadora Independiente; Guayaquil, Ecuador; draintriago@live.com; (D) https://orcid.org/0000-0001-82444286

3. Médico; Investigador Independiente; Guayaquil, Ecuador; carlos_villacis90@hotmail.com; (iD https://orcid.org/0000-00018842-2707

4. Master Universitario en Prevención de Riesgos Laborales; Médico; Investigador Independiente; Guayaquil, Ecuador; jeffo. leo.grc@gmail.com; iD https://orcid.org/0000-0003-3383-0783

\section{CORRESPONDENCIA}

Pedro Enrique Decker Larrea

pedro_deckerbond@hotmail.com

Guayaquil, Ecuador 


\section{RESUMEN}

La hipertensión intracraneal idiopática $(\mathrm{HII})$ es un síndrome caracterizado por un aumento de la presión intracraneal de causa desconocida, que conduce a dolor de cabeza intenso, papiledema y alteraciones visuales. Su nombre anterior, pseudotumor cerebri, ha ganado popularidad recientemente. Los factores de riesgo más fuertes y consistentes se presentan en pacientes de sexo femenino y en aquellos que padecen de obesidad. Con poca frecuencia, la HII puede presentarse en ausencia de papiledema mostrando un perfil de cefalea similar a la cefalea crónica diaria con características migrañosas. Se han propuesto varios mecanismos para explicar la etiología de este trastorno asociado con diversas condiciones clínicas. En los últimos años, se han presentado algunos factores inflamatorios, péptidos natriuréticos y acuaporinas como posibles contribuyentes de la patogenia. Por otro lado, algunos investigadores han informado que la estenosis del seno transverso bilateral se observa en la mayoría de los pacientes con HII; por lo tanto, en algunos pacientes se utiliza la colocación de un stent de seno dural. Ninguna teoría ha sido capaz de proporcionar una respuesta completa y no existe consenso sobre la causa exacta de la HII. El objetivo de esta revisión fue discutir los nuevos conocimientos sobre la misteriosa patogénesis de la IIH.

Palabras clave: Hipertensión Intracraneal Idiopática; Pseudotumor Cerebri; Estenosis Del Seno Transverso.

\section{ABSTRACT}

Idiopathic intracranial hypertension $(\mathrm{IIH})$ is a syndrome characterized by an increase in intracranial pressure of unknown cause, leading to severe headache, papilledema, and visual disturbances. Its former name, pseudotumor cerebri, has recently gained popularity. The strongest and most consistent risk factors occur in female patients and those with obesity. Infrequently, IIH can present in the absence of papilledema showing a headache profile similar to chronic daily headache with migraine characteristics. Several mechanisms have been proposed to explain the etiology of this disorder associated with various clinical conditions. In recent years, some inflammatory factors, natriuretic peptides, and aquaporins have been reported as possible contributors to pathogenesis. On the other hand, some investigators have reported that bilateral transverse sinus stenosis is seen in most patients with $\mathrm{IIH}$; therefore, dural sinus stenting is used in some patients. No theory has been able to provide a complete answer and there is no consensus on the exact cause of IIH. The aim of this review was to discuss the new insights into the mysterious pathogenesis of $\mathrm{IH}$.

Keywords: Idiopathic Intracranial Hypertension; Pseudotumor Cerebri; Transverse Sinus Stenosis.

\section{RESUMO}

A hipertensão intracraniana idiopática (HII) é uma síndrome caracterizada por um aumento da pressão intracraniana de causa desconhecida, levando a cefaleia intensa, papiledema e distúrbios visuais. Seu nome anterior, pseudotumor cerebri, ganhou popularidade recentemente. Os fatores de risco mais fortes e consistentes ocorrem em pacientes do sexo feminino e com obesidade. Raramente, a HII pode se manifestar na ausência de papiledema, mostrando um perfil de cefaleia semelhante à cefaleia diária crônica com características de enxaqueca. Vários mecanismos têm sido propostos para explicar a etiologia desse distúrbio associada a várias condições clínicas. Nos últimos anos, alguns fatores inflamatórios, peptídeos natriuréticos e aquaporinas foram relatados como possíveis contribuintes para a patogênese. Por outro lado, alguns pesquisadores relataram que a estenose do seio transverso bilateral é observada na maioria dos pacientes com HII; portanto, o implante de stent do seio dural é usado em alguns pacientes. Nenhuma teoria foi capaz de fornecer uma resposta completa e não há consenso sobre a causa exata da HII. O objetivo desta revisão foi discutir os novos insights sobre a misteriosa patogênese da HII.

Palavras-chave: Hipertensão Intracraniana Idiopática; Pseudotumor Cerebral; Estenose Do Seio Transverso. 


\section{Introducción}

La hipertensión intracraneal idiopática (HII) es una entidad clínica bien conocida pero poco investigada con antecedentes fisiopatológicos no resueltos; por lo tanto, su diagnóstico y manejo óptimo generalmente crea problemas para los médicos. Los términos "pseudotumor cerebral" e "hipertensión intracraneal benigna" se aplicaron originalmente a pacientes con aumento de la presión intracraneal (PIC) en los que no se encontró ningún tumor y cuyo curso se creía que era benigno.

Sin embargo, "este cuadro clínico no siempre es benigno ni está relacionado con un tumor falso y, por lo tanto, su terminología se cambió a hipertensión intracraneal idiopática" (Cigeroglu, Gokyigit, \& BaykanKurt, 1994). Por otro lado, Friedman, Liu, \& Digre, (2013) recientemente propusieron que "el término general pseudotumor cerebral debería usarse para los pacientes que tienen PIC con una etiología poco clara o causas secundarias, como medicamentos y otras afecciones médicas". La patogenia de la PIC elevada en estas condiciones aún no está clara. Se han postulado varios mecanismos fisiopatológicos que regulan la presión del líquido cefalorraquídeo (LCR). En esta revisión, el objetivo es discutir los nuevos conocimientos emergentes sobre la patogénesis de la $\mathrm{IIH}$.

La incidencia de HII es de 1 a 2 en 100.000 habitantes, pero aumenta a 19 en 100.000 mujeres obesas en edad fértil. Existe una clara predilección por las mujeres sobre los hombres que varía de 4,3:1 a 15:1 en la literatura. "La HII también puede ocurrir en niños, pero la obesidad y el predominio femenino parecen ser menos comunes en los niños prepúberes con HII" (Giovanna \& Piero, 2017)

La HII se caracteriza por una PIC que produce cefalea, papiledema, síntomas y signos visuales, sin hallazgos de lateralización en el examen neurológico y hallazgos normales del LCR. El dolor de cabeza atribuido a HIl debe ser progresivo con al menos una de las siguientes características; aparición diaria, difusa y / o constante (no pulsante) y agravada por la tos o el esfuerzo.Con poca frecuencia, la HII puede presentarse en ausencia de edema de papila y los pacientes pueden experimentar un perfil de dolor de cabeza similar al dolor de cabeza diario crónico con características migrañosas, que también responden a agentes antimigrañosos específicos

Existen algunas diferencias clínicas intrigantes con respecto al resultado visual en pacientes mayores y hombres con HII. "Los pacientes mayores de 50 años presentan menos quejas de dolor de cabeza y alteraciones visuales y se ha evidenciado que el pronóstico visual es mejor en los ancianos" (Bruce, Kedar, Van Stavern, \& Corbett, 2010). También "hay alguna evidencia de que los pacientes con HII tienen cambios en el volumen del bulbo olfatorio y disminución del olfato que acompañan a otros síntomas convencionales de HII" (Kunte, Schmidt, \& Kronenberg, 2013).

No obstante, los hombres con HIl deben ser seguidos más de cerca con respecto a la función visual porque es posible que no experimenten o notifiquen otros síntomas de HII como dolor de cabeza que alarme al médico. En los niños con HII, "el dolor de cabeza es el síntoma más común, pero también tienen una mayor incidencia de disfunción de la motilidad ocular y los déficits de los nervios craneales son más comunes en comparación con los pacientes adultos" (Giovanna \& Piero, 2017).

En el siguiente proceso investigativo se presenta un análisis bibliográfico de la fisiopatología y el manejo de esta enfermedad con el objetivo de brindar información de interés que sirva para futuras investigaciones.

\section{Metodología}

Para el desarrollo de este proceso investi 
gativo, se plantea como metodología la encaminada hacia una orientación científica particular que se encuentra determinada por la necesidad de indagar en forma precisa y coherente una situación, en tal sentido Davila, (2015) define la metodología "como aquellos pasos anteriores que son seleccionados por el investigador para lograr resultados favorables que le ayuden a plantear nuevas ideas" (p.66)

Lo citado por el autor, lleva a entender que el desarrollo de la acción investigativa busca simplemente coordinar acciones enmarcadas en una revisión bibliográfica con el fin de complementar ideas previas relacionadas Hipertensión intracraneal idiopática, fisiopatología y manejo a través de una revisión de literatura, para así finalmente elaborar un cuerpo de consideraciones generales que ayuden a ampliar el interés propuesto.

\section{Tipo de Investigación}

Dentro de toda práctica investigativa, se precisan acciones de carácter metodológico mediante las cuales se logra conocer y proyectar los eventos posibles que la determinan. En este sentido, la presente investigación corresponde al tipo documental, definido por Castro (2016), "se ocupa del estudio de problemas planteados a nivel teórico, la información requerida para abordarlos se encuentra básicamente en materiales impresos, audiovisuales y / o electrónicos". (p.41).

En consideración a esta definición, la orientación metodológica incluye la oportunidad de cumplir con una serie de actividades inherentes a la revisión y lectura de diversos documentos, donde se encuentran ideas explicitas relacionadas con los tópicos encargados de identificar una característica inmersa en el estudio. Por lo tanto, se realizaron continuas interpretaciones con el claro propósito de revisar aquellas apreciaciones propuestas por diferentes investigadores en relación al tema de interés, para luego dar la respectiva argumentación a los planteamientos, en función a las necesidades encontradas en la investigación, apoyados en las herramientas tecnológicas para la búsqueda de trabajos con valor científico disponibles en la web que tenían conexión con el objetivo principal de la investigación.

\section{Fuentes Documentales}

El análisis correspondiente a las características que predomina en el tema seleccionado, llevan a incluir diferentes fuentes documentales encargadas de darle el respectivo valor científico y en ese sentido cumplir con la valoración de los hechos a fin de generar nuevos criterios que sirven de referencia a otros procesos investigativos. Para Castro,(2016) las fuentes documentales incorporadas en la investigación documental o bibliográfica, "representa la suma de materiales sistemáticos que son revisados en forma rigurosa y profunda para llegar a un análisis del fenómeno" (p.41). Por lo tanto, se procedió a cumplir con la lectura previa determinada para encontrar aquellos aspectos estrechamente vinculados con el tema, con el fin de explicar mediante un desarrollo las respectivas apreciaciones generales de importancia.

\section{Técnicas para la Recolección de la Infor- mación}

La conducción de la investigación para ser realizada en función a las particularidades que determinan a los estudios documentales, tiene como fin el desarrollo de un conjunto de acciones encargadas de llevar a la selección de técnicas estrechamente vinculadas con las características del estudio. Bolívar, (2015), refiere, que es "una técnica particular para aportar ayuda a los procedimientos de selección de las ideas primarias y secundarias". (p.71).

Tal como lo expresa, Bolívar, (2015) "Las técnicas documentales proporcionan las

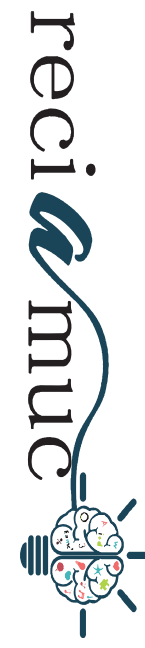


herramientas esenciales y determinantes para responder a los objetivos formulados y llegar a resultados efectivos" (p. 58). Es decir, para responder con eficiencia a las necesidades investigativas, se introdujeron como técnica de recolección el método inductivo, que hizo posible llevar a cabo una valoración de los hechos de forma particular para llegar a la explicación desde una visión general. El autor Bolívar, (2015) tambien expresa que las técnicas de procesamiento de datos en los estudios documentales "son las encargadas de ofrecer al investigador la visión o pasos que deben cumplir durante su ejercicio, cada una de ellas debe estar en correspondencia con el nivel a emplear" (p. 123). Esto indica, que para llevar a cabo el procesamiento de los datos obtenidos una vez aplicadas las técnicas seleccionadas, tales como: fichas de resumen, textual, registros descriptivos entre otros, los mismos se deben ajustar al nivel que ha sido seleccionado.

\section{Resultados}

\section{Fisiopatología}

Aunque se reconoce desde hace más de un siglo, la patogenia de la HII aún no está clara. Hay varios mecanismos propuestos como edema parenquimatoso, aumento del volumen sanguíneo cerebral, producción excesiva de LCR; obstrucción del flujo de salida venoso y resorción comprometida del LCR. En años recientes, también se ha propuesto la posible contribución de factores inflamatorios. Ninguna teoría por sí sola ha podido proporcionar una respuesta completa y no existe consenso sobre la causa exacta de la HII.

Los autores (Esqueda, Gutiérrez, \& Cuéllar, 2014) informaron "evidencia microscópica de edema intracelular y extracelular, luego estudios de RM mostraron mayor contenido de agua en el cerebro y difusión de agua en la sustancia blanca subcortical, lo que proporciona una evidencia indirecta de edema cerebral sin confirmación adicional". Entrando un poco en historia, Dandy, (1937) "primero planteó la hipótesis de que el volumen de sangre cerebral o de LCR podría aumentar en esta afección". Sumado a esto, "algunos informes posteriores apoyaron la presencia de un aumento del volumen sanguíneo cerebral en la HII mediante la inyección de un marcador intracarotídeo en pacientes con HII anestesiados" (Raichle \& Grubb, 1978). También se ha propuesto una producción excesiva de LCR, pero el tamaño ventricular normal o disminuido encontrado en los pacientes con HII sugiere que no hay un aumento de la producción de LCR en la mayoría de los pacientes.

\section{Obesidad e inflamación crónica}

Los factores de riesgo más fuertes y consistentes de HII son la obesidad y el sexo femenino; al igual que las mujeres, los hombres con HIl también suelen ser obesos. Los autores Daniels, Liu, \& Volpe, (2007) demostraron "una correlación entre el índice de masa corporal (IMC) y el riesgo de HII y se encontró que el aumento de peso estaba asociado con la recurrencia de la enfermedad". Además, los pacientes con HII severamente obesos tenían un papiledema más avanzado y una pérdida visual más grave que los pacientes menos obesos, e incluso una reducción de peso relativamente pequeña ha mejorado la visión según algunos estudios.

Aunque la obesidad está claramente asociada con HII, su mecanismo fisiopatológico permanece indeterminado. Se ha sugerido que la obesidad que causa un aumento de la presión intraabdominal podría conducir a una reducción del drenaje venoso cerebral.

La inflamación crónica asociada con la obesidad, que puede conducir a un estado protrombótico, se ha propuesto como uno de los posibles factores etiológicos en el desarrollo de la HII. La obesidad se reconoce como un estado proinflamatorio y se asocia 
con un aumento de la expresión de varias adipocinas y diversas citocinas, incluidas leptina e interleucinas, proteína quimiotáctica de macrófagos-1 (MCP-1 / CCL2) e inhibidor del activador del plasminógeno-1 (PAl-1). Se encuentra que los niveles de leptina fluctúan durante un estado inflamatorio. (Daniels, Liu, \& Volpe, 2007)

El tejido adiposo se considera un tejido endocrino secretor activo. Una de las hipótesis de larga data sobre la patogenia de la HII es el metabolismo anormal de la vitamina A. De acuerdo con Warner, Larson, \& Bhosale, (2007) estudios mostraron que el "nivel de retinol está elevado en el LCR de pacientes con HII. La proteína de unión al retinol derivada del tejido adiposo se libera del tejido adiposo y actúa como un modulador de la sensibilidad a la insulina". Dada la fuerte predilección de la IIH por las mujeres jóvenes obesas, se necesitan estudios más amplios para dilucidar el verdadero papel del LCR frente a la leptina sérica y otras citocinas y moléculas producidas por el tejido adiposo en la fisiopatología de la HII.

\section{Disfunción hormonalsexual}

Se sospecha que las hormonas sexuales tienen una función en la patogenia de la HII debido a la ocurrencia preferencial del trastorno entre mujeres pospúberes y premenopáusicas y la ausencia de una preferencia de género antes de la pubertad. A pesar de algunos informes contradictorios, no se puede descartar por completo el papel de una alteración de las hormonas sexuales en la patogénesis. "Muchas pacientes tienen antecedentes de irregularidades menstruales y se han evidenciado informes de casos que relacionan la HII con el uso de píldoras anticonceptivas orales y también con la enfermedad de ovario poliquístico" (Glueck, lyengar, \& Goldenberg, 2003). El síndrome de ovario poliquístico (SOP) es una afección endocrina crónica que conduce a la disfunción de la menstruación y la ovulación, relacionada con la obesidad, altos niveles de leptina sérica e inflamación de bajo grado.

El tratamiento de primera línea es la reducción de peso, que también es valiosa en el tratamiento de la IIH. Se informa que la prevalencia del SOP en mujeres derivadas debido a HII es de $39 \%$ a $57 \%$. Por lo tanto, la proporción es de 5 a 8 veces mayor que la prevalencia del $7 \%$ de SOP en la población general no seleccionada. Además, se encontró que la HII está particularmente asociada con la obesidad de tipo ginecoide más que con la obesidad abdominal, lo que también enfatiza la función de la disfunción hormonal sexual en la patogenia de la HII (Kesler, Kliper, Shenkerman, \& Stern, 2010).

\section{Péptidos natriuréticos}

Este sistema de péptidos natriuréticos que también se expresa en el sistema nervioso central comprende "una familia de neuropéptidos (NP) estructuralmente relacionados con propiedades antagonistas contra el sistema renina-angiotensina-aldosterona" (Gamboa \& Vivas, 2002). "El péptido natriurético auricular (ANP) y el péptido natriurético tipo B (BNP) más conocidos se liberan principalmente de los cardiomiocitos en respuesta al aumento de la tensión de la pared y promueven la nutriereis y la diuresis" (Gamboa \& Vivas, 2002).

Por el contrario, el péptido natriurético de tipo C (CNP) se libera de varios tejidos, incluidas las células endoteliales, y actúa como un relajante paracrino del tono vascular. Se ha demostrado un aumento de las concentraciones de NP en el LCR en la hipertensión intracraneal, es decir, hemorragia subaracnoidea. Además, la administración intraventricular de ANP reduce la producción elevada de PIC y LCR en modelos de roedores.

Dado que dos de estos tres receptores NP, NPR-A y NPR-C, se han ubicado en el plexo coroideo donde se generan dos tercios de la producción total de LCR, se ha sugerido 
que las NP pueden estar involucradas en la regulación de la dinámica del licor.

Los niveles plasmáticos de proCNP fueron significativamente más bajos en los pacientes con IIH y los niveles de proBNP fueron significativamente más bajos en los pacientes con IIH en comparación con los de los controles. Más interesante aún, las concentraciones plasmáticas de estos neuropéptidos se relacionan inversamente con el IMC y pueden aumentar durante la pérdida de peso (Skau, Goetze, Rehfeld, \& Jensen, 2010).

Teniendo en cuenta la asociación entre la producción periférica de NP y la obesidad, y las acciones reguladoras de la PIC propuestas por las NP, es tentador plantear la hipótesis de que existe un vínculo entre las concentraciones de IIH y NP.

\section{Acuaporinas y anticuerpos anti neurona- les}

El descubrimiento de la familia de acuaporinas (AQP) de canales de agua de membrana ha proporcionado nuevos conocimientos sobre la fisiopatología de la homeostasis del agua en el cerebro. "Los AQP son una gran familia de canales de agua, expresados en la membrana plasmática de muchos tipos de células en el SNC y el ojo" (López \& Rodríguez, 2019).

La acuaporina 1 (AQP1) se detecta principalmente en las células epiteliales del plexo coroideo, responsable de la secreción de agua en el espacio subaracnoideo.

Se podrían usar medicamentos para la HII para regular a la baja la AQP1 y se ha demostrado que varios fármacos como los retinoides y los esteroides inducen la expresión de AQP1, lo que sugiere la función causal de AQP1 en la patogénesis de la HII inducida por fármacos. Además, se encontró AQP1 estar asociado con el aumento de peso en un modelo animal (Ma, Jayaraman, \& Wang, 2001).
La acuaporina-4 (AQP4) es otro de los principales canales de agua del cerebro. "Se presume que desempeña un papel funcional importante en el transporte de agua dentro y fuera del cerebro" (López \& Rodríguez, 2019), debido a su amplia distribución dentro del SNC, que incluye el plexo coroideo y las células ependimarias de los ventrículos y su localización crítica en los procesos astrocíticos del pie a lo largo de la barrera hematoencefálica y la interfaz cerebro-LCR.

\section{Nuevos conocimientos de historia de la estenosis del seno transverso bilateral}

La neuroimagen y el diagnóstico de HII se basan en la neuroimagen normal del cerebro, incluido el MRV. Sin embargo, existe evidencia actual de que algunos pacientes con HII tienen estenosis del seno transverso bilateral (BTSS). Se sabe que "los senos transversales son asimétricos en la mayoría de los individuos y un seno transverso hipoplásico unilateral se considera una "variante normal" sin ningún cambio informado de la PIC" (Agarwal, Kumar, \& Arora, 2010). La MRV con una secuencia ordenada céntrico elíptico auto activado demostró grados variables de estenosis venosa cerebral en la mayoría de los casos de HII en comparación con los controles normales.

Además, se encontró que el BTSS predice la presencia de aumento de la PIC en pacientes diagnosticados con migraña $\mathrm{O}$ cefalea tensional crónica. Por lo tanto, se ha propuesto que la presencia de estenosis sinusal transversa puede respaldar el diagnóstico de HII sin papiledema, como se mencionó anteriormente. Pero, BTSS se encuentra con poca frecuencia en la práctica clínica, probablemente dependiendo de la técnica de imagen y la experiencia del neurorradiólogo (Agarwal, Kumar, \& Arora, 2010) (Figura 1).

A continuación se presenta una imagen donde se observa una estenosis prominente en el transverso izquierdoseno e irregularidades en el seno transverso derecho. 
Figura 1. Venografía por RM estándar de un paciente con diagnóstico de HII

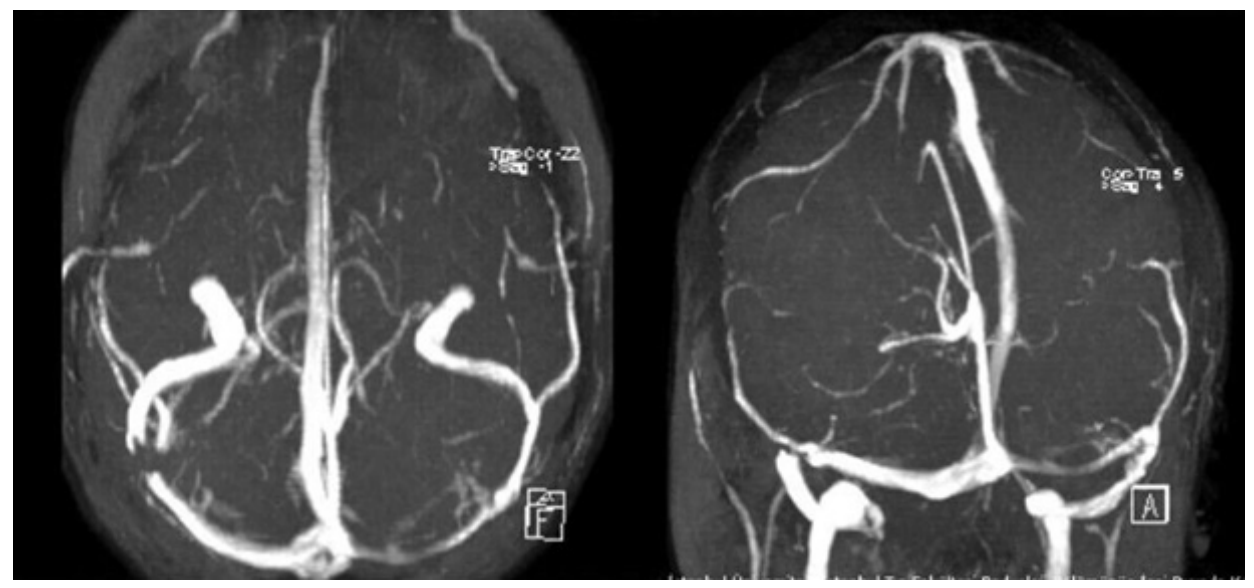

Fuente. (Agarwal, Kumar, \& Arora, 2010).

Sin embargo, existe un debate sobre si tal estenosis es la causa o el efecto de la $\mathrm{IIH}$. Algunos autores argumentaron que la teoría del seno venoso no podía explicar el predominio femenino de HII en adultos. Además, el curso clínico, incluida la pérdida del campo visual, no se correlacionó con el grado de estenosis del seno transverso. Se ha sugerido que tal estrechamiento "es secundario a la compresión de los senos nasales por la PIC elevada que causa obstrucción del flujo de salida, lo que da como resultado una mayor hipertensión venosa, luego disminuye la absorción de LCR y provoca más PIC" (Stevens, Previte, \& Lakin, 2007). Adicionalmente, algunos estudios anatómicos sugirieron

Que estas estenosis se deben a la presencia de trabéculas, septos o granulaciones hipertrofiadas en los senos transversales. También se propuso un mecanismo de retroalimentación positiva que sugiere que la hipertensión venosa conduce a aumentó aún más la PIC, independientemente de la causa inicial de la estenosis focal (Puffer, Mustafa, \& Lanzino, 2013)

Por tanto, en algunos pacientes se utilizó la endoprótesis de seno venoso con resultados contradictorios. Algunos investigadores encontraron que la colocación de un stent en el seno transverso es eficaz en los pa- cientes con HII y sugirieron que podría ser una nueva opción de tratamiento en este grupo.

Es evidente que muchos hallazgos sugieren que las alteraciones del flujo venoso en la HII son probablemente la consecuencia de la hipertensión del LCR, pero no su causa. Sin embargo, la colocación de un stent en el seno transverso puede evaluarse como una opción de tratamiento eficaz rompiendo el ciclo de biorretroalimentación positiva anormal anteriormente mencionado, en pacientes seleccionados que tienen HII médicamente refractaria.

\section{Causas secundarias, factores subyacen- tes}

La presión intracraneal (PIC) puede ser secundaria a varias causas, como trombosis del seno venoso, afecciones médicas y fármacos. Agarwal, Kumar, \& Arora, (2010) propusieron que "el aumento de presión venosa podría ser el factor clave en el desarrollo de HIl porque es el mecanismo unificador de todos los síndromes benignos similares a tumores. Se encontró trombosis venosa cerebral en $11,4 \%$ de pacientes que tenían HII" (p. 153)

La HII se ha asociado con muchas etiolo-

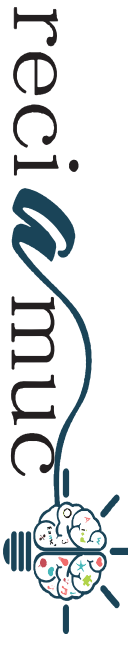


gías, como la exposición a varios fármacos (vitamina A, hormona del crecimiento, esteroides, minociclina y tetraciclina, sulfasalazina, etc.).

Enfermedad de Behçet, malformaciones arteriovenosas, trastornos del sueño, incluido el síndrome de apnea obstructiva del sueño (SAOS), hipertensión venosa extracraneal secundaria a defecto del tabique cardíaco, lupus eritematoso sistémico, uremia, anemia ferropénica, así como algunos trastornos endocrinos cambios tales como irregularidades menstruales, uso de anticonceptivos orales, hipertiroidismo e hipotiroidismo (Press \& Ladenson, 2013)

También se ha argumentado que un defecto trombolítico subyacente en pacientes con HII podría desempeñar un papel en la patogenia y algunos estudios pequeños mostraron anomalías en los factores protrombóticos. Teóricamente, al menos en algunos pacientes, "la HII podría deberse a micro trombos que impiden el drenaje del LCR, pero no demostrable en las imágenes. Por lo tanto, el papel de los factores trombóticos en la HII debe investigarse mediante estudios más amplios" (Press \& Ladenson, 2013).

\section{Opciones de tratamiento}

En la actualidad, la HII no cuenta con un algoritmo de manejo bien establecido, dada la escasez de estudios sobre el tema. La dinámica y la homeostasis del LCR en la HII son complejas y no se comprenden completamente. Se anima a los pacientes obesos a perder peso y la acetazolamida es la primera opción de tratamiento médico. El objetivo principal es disminuir la PIC para preservar la función visual y eliminar el dolor de cabeza. En los pacientes con HII que todavía tienen dolor de cabeza después de la resolución de la PIC, "en la práctica clínica se utilizan medicamentos preventivos para el dolor de cabeza como el topiramato, que también tiene una actividad leve de anhidrasa carbónica" (Celebisoy \& Gökçay, 2007).

El papel terapéutico de las punciones lumbares repetidas que fueron populares en el pasado ahora se cuestiona debido a sus dificultades para el paciente y la falta de evidencia que demuestre sus efectos duraderos. Las opciones quirúrgicas utilizadas en casos seleccionados con pérdida visual incluyen la fenestración de la vaina del nervio óptico, procedimientos de derivación del LCR que incluyen derivación lumboperitoneal o ventriculoperitoneal, además de la cirugía bariátrica para la obesidad y la implantación de un stent sinusal transverso recién introducido como se discutió anteriormente. La HII suele ser una enfermedad autolimitante, pero muestra recaídas en algunos de los pacientes afectados.

\section{Conclusión}

La patogenia de la Hipertension Intracraneal Idiopatica (HII) aún no se comprende completamente. Es probable que contribuyan a la patogenia múltiples mecanismos coexistentes, incluida la producción excesiva de líquido cefalorraquídeo (LCR), la resorción deficiente de LCR y la obstrucción del flujo venoso de salida.

Durante el desarrollo de la investigación se observó, que el aumento de peso claramente juega un papel en el proceso de la enfermedad, tomando en consideración que la presión del LCR y los síntomas de HII tienden a fluctuar con remisiones espontáneas (a veces de forma permanente), que son más favorables a un proceso inflamatorio. La estenosis del seno transverso bilateral (BTSS) puede ser un marcador de aumento de la presión intracraneal (PIC), pero aún no se ha resuelto si es la causa o el resultado de la HII. Se necesitan estudios futuros para optimizar la comprensión biológica y las opciones de tratamiento razonables de esta intrigante enfermedad. 


\section{Bibliografía}

Agarwal, P., Kumar, M., \& Arora, V. (2010). Clinical profile of cerebral venous sinus thrombosis and the role of imaging in its diagnosis in patients with presumed idiopathic intracranial hypertension. Indian J Ophthalmol , 153-5.

Bruce, B., Kedar, S., Van Stavern, G., \& Corbett, J. (2010). Atypical idiopathic intracranial hypertension: normal BMI and older patients. Neurology , 1827-32.

Celebisoy, N., \& Gökçay, F. (2007). Treatment of idiopathic intracranial hypertension: topiramate vs acetazolamide, an open-label study. Acta Neurol Scand, 322-7.

Cigeroglu, O., Gokyigit, A., \& Baykan-Kurt, B. (1994). Pseudotumour cerebri: Review of 34 cases. (in Turkish). Ar-chives of Neuropsychiatry , 57-62.

Dandy, W. (1937). Intracranial pressure without brain tumor: diagnosis and treatment. Ann Surg, 492513

Daniels, A., Liu, G., \& Volpe, N. (2007). Profiles of obesity, weight gain, and quality of life in idiopathic intracranial hypertension (pseudotumor cerebri). Am J Ophthalmol , 635-41.

Esqueda, M., Gutiérrez, J., \& Cuéllar, S. (2014). Edema cerebral I: fisiopatología, manifestaciones clínicas, diagnóstico y monitoreo neurológico. Med Int Méx , 30, 584-590.

Friedman, D., Liu, G., \& Digre, K. (2013). Revised diagnostic criteria for the pseudotumor cerebri syndrome in adults and children. Neurology , 115965.

Gamboa, R., \& Vivas, P. (2002). LOS PÉPTIDOS NATRIURÉTICOS Y SU EFECTO CARDIOVASCULAR. Revista Peruana de Cardiologia , 28 (1).

Giovanna, V., \& Piero, P. (2017). Therapeutic approaches to pediatric pseudotumor cerebri: New insights from literature data. Int J Immunopathol Pharmacol. , 1 (30), 94-97.

Glueck, C., lyengar, S., \& Goldenberg, N. (2003). Idiopathic intracranial hypertension: associations with coagulation disorders and polycysticovary syndrome. J Lab Clin Med, 35-45.

Kesler, A., Kliper, E., Shenkerman, G., \& Stern, N. (2010). Idiopathic intra-cranial hypertension is associated with lower body adipos-ity. Ophthalmology , 169-74.

Kunte, H., Schmidt, F., \& Kronenberg, G. (2013). Olfactory dysfunction in patients with idio-pathic in- tracranial hypertension. Neurology , 379-82.

López, S., \& Rodríguez, C. (2019). Las Aquaporinas Y El Sistema Nervioso Central. Revisa Ecuatoriana de Neurologia , 28 (2).

Ma, T., Jayaraman, S., \& Wang, K. (2001). Defective dietary fat processing in transgenic mice lack-ing aquaporin-1 water channels. Am J Physiol Cell Physiol, 126-34.

Press, O., \& Ladenson, P. (2013). Pseudotumor cerebri and hypothyroidism. Arch Intern Med, 167-8.

Puffer, R., Mustafa, W., \& Lanzino, G. (2013). Venous sinus stenting for idiopathic intracranial hypertension: a review of the litera-ture. J Neurointerv Surg , 483-6.

Raichle, M., \& Grubb, R. J. (1978). Cerebral hemodynamics and metabolism in pseudotumor cerebri. Ann Neurol , 104-11.

Skau, M., Goetze, J., Rehfeld, J., \& Jensen, R. (2010). Natriuretic propeptides in idiopathic intracranial hypertension. Regul Pept, 71-7.

Smith, J. (1985). Whence pseudotumor cerebri? . J Clin Neuroophthalmol , 55-6.

Stevens, S., Previte, M., \& Lakin, W. (2007). Idiopathic intracranial hypertension and transverse sinus stenosis: a modelling study. Math Med Biol , 85109.

Warner, J., Larson, A., \& Bhosale, P. (2007). Retinol-binding protein and retinol analysis in ce-rebrospinal fluid and serum of patients with and without idiopathic intracranial hypertension. J Neuroophthalmol , 258-62. 
